\title{
Tapentadol extended-release for treatment of chronic pain: a review
}

This article was published in the following Dove Press journal:

Journal of Pain Research

29 July 2011

Number of times this article has been viewed

\author{
Nalini Vadivelu' \\ Alexander Timchenko' \\ Yili Huang ${ }^{2}$ \\ Raymond Sinatra'
}

'Department of Anesthesiology, Yale University School of Medicine, New Haven, CT; ${ }^{2}$ Internal Medicine, North Shore-LIJ Plainview Hospital, Plainview, NY, USA
Correspondence: Nalini Vadivelu Department of Anesthesiology, Yale University School of Medicine, 333 Cedar Street, New Haven, CT 06520, USA

Tel +l 2047852802

Fax + I 2037858884

Email nalini.vadivelu@yale.edu
Abstract: Tapentadol is a centrally acting analgesic with a dual mechanism of action of mu receptor agonism and norepinephrine reuptake inhibition. Tapentadol immediate-release is approved by the US Food and Drug Administration for the management of moderate-to-severe acute pain. It was developed to decrease the intolerability issue associated with opioids. Tapentadol extended-release has a 12-hour duration of effect, and has recently been evaluated for pain in patients with chronic osteoarthritis, low back pain, and pain associated with diabetic peripheral neuropathy. Tapentadol extended-release was found to provide safe and highly effective analgesia for the treatment of chronic pain conditions, including moderate-to-severe chronic osteoarthritis pain and low back pain. Initial trials demonstrating efficacy in neuropathic pain suggest that tapentadol has comparable analgesic effectiveness and better gastrointestinal tolerability than opioid comparators, and demonstrates effectiveness in settings of inflammatory, somatic, and neuropathic pain. Gastrointestinal intolerance and central nervous system effects were the major adverse events noted. Tapentadol will need to be rigorously tested in chronic neuropathic pain, cancer-related pain, and cancer-related neuropathic pain.

Keywords: osteoarthritis, neuropathic pain, analgesic, opioids, norepinephrine

\section{Background}

Centrally acting analgesics with a dual mechanism of action have recently been developed in an attempt to reduce the tolerability issues associated with opioids while providing equivalent analgesia, and are being evaluated for the treatment of chronic pain. Tapentadol is a novel centrally acting analgesic, initially formulated as an immediate-release preparation. It is a potent Schedule II analgesic approved for use by the US Food and Drug Administration (FDA) in 2009, and is the first pain reliever developed in over 25 years for the management of moderate-to-severe acute pain.

Tapentadol immediate-release is available as 50,75, and $100 \mathrm{mg}$ tablets and provides 4-6 hours of analgesia. Tapentadol immediate-release was shown to provide analgesia comparable with that of $10-15 \mathrm{mg}$ of immediate-release oxycodone ${ }^{1,2}$ in patients recovering from dental extraction pain ${ }^{3}$ and pain following bunionectomy. ${ }^{46}$ It was also as effective as oxycodone in patients presenting with chronic osteoarthritis pain and chronic low back pain. ${ }^{7,8}$ Of importance in the comparator trials was the finding that patients treated with tapentadol had a lower incidence of adverse gastrointestinal events, including nausea, vomiting, and constipation, than those treated with oxycodone. In a bunionectomy trial, the composite incidence of nausea and vomiting in patients treated with tapentadol $50 \mathrm{mg}$ every 6 hours was significantly lower than in patients treated with oxycodone $10 \mathrm{mg}$. Based on the safety and efficacy profile of the immediate-release 
preparation, the Pricara division of Johnson and Johnson Pharmaceuticals made the decision to develop a preparation that provided a more prolonged duration of effect in an indication of chronic pain. The new preparation, tapentadol extended-release (ER), is a sustained-release tablet with a 12-hour duration of effect for patients 18 years of age and older with moderate-to-severe chronic pain who require around the clock analgesic therapy.

At the time of writing, clinical development of tapentadol ER has been completed, submitted, and reviewed by the FDA. The submission included a number of large-scale, randomized, active comparator-controlled and placebo-controlled Phase III studies that evaluated the safety and efficacy of tapentadol in patients with chronic osteoarthritis, low back pain, or pain associated with diabetic peripheral neuropathy. Tapentadol ER has also been evaluated in a 1-year, activecontrolled, open-label Phase III safety trial. The FDA has not requested additional efficacy trials, but in a complete response letter provided on November 2010, the agency seeks additional information regarding modifications to the ER tablet that increase mechanical resistance to breaking or crushing prior to granting approval for use. In a series of largescale, controlled clinical trials, tapentadol ER was found to provide safe and highly effective analgesia for the treatment of chronic pain conditions, including moderate-to severe chronic osteoarthritis pain and lower back pain. ${ }^{1,2,4}$ Like the immediate-release preparation, it provides potent analgesia equivalent or superior to that of sustained-release opioids with an unexpected reduction in adverse gastrointestinal events. It also appears to have analgesic effectiveness comparable with that of opioid comparators, demonstrating effectiveness in settings of inflammatory, somatic, and neuropathic pain.

\section{Pharmacology}

Tapentadol produces potent analgesic effects via its dual mechanism of action, ie, mu receptor agonism and norepinephrine reuptake inhibition..$^{9-12}$ In animal models, tapentadol behaves as a weak opioid agonist, with 50 times less affinity than morphine for the mu receptor. Despite this low binding affinity, tapentadol provides powerful analgesic effects, equivalent to one third that observed with equianalgesic doses of morphine., ${ }^{2,13}$ This finding and observations noted in Phase I human trials suggested that mu receptor activation provides a measurable but limited contribution to the analgesic effect of tapentadol.

Norepinephrine inhibits transmission of noxious impulses by activating alpha adrenergic receptors located on noxious nerve fibers in the spinal cord and central nervous system. Tapentadol has high selectivity for the norepinephrine transporter protein, and blocks reuptake of norepinephrine at the terminal endings of interneurons and descending inhibitory fibers. The resulting increase in norepinephrine further suppresses pain transmission at nociceptor endings. Unlike tramadol, which inhibits reuptake of both norepinephrine and serotonin, tapentadol has limited interaction with serotonin transporter proteins and minimal effect upon serotonin reuptake. ${ }^{1,2,13}$ Therefore, the risk of serotonin syndrome is less with tapentadol. Moreover, it was suggested that analgesia is more readily obtained by norepinephrine reuptake inhibition than by serotonin inhibition. ${ }^{14,15}$ From preliminary anecdotal information, it appears that treboxetine does not provide good analgesia. In addition, it has been seen that tricyclic antidepressants with more norepinephrine reuptake inhibition than serotonin reuptake inhibition do not appear to possess significantly more potent analgesic qualities than tricyclic antidepressants with more serotonin inhibition than norepinephrine reuptake inhibition.

Tapentadol exists as a single active enantiomer ${ }^{1,2,13}$ and is metabolized mainly by O-glucuronidation. Its principal metabolite is inactive, having no affinity for the mu receptor or the norepinephrine transporter. Because the analgesic activity of tapentadol is limited to the primary molecule, no enzymes are needed to convert it to an active metabolite, as is the case for tramadol and codeine. The development of tapentadol proceeded from the analgesic with a combined mechanism of action, ie, tramadol. ${ }^{16,17}$ Tramadol provides weak mu activation with inhibition of serotonin and norepinephrine uptake. ${ }^{18-20}$ The pharmacokinetic properties of tapentadol differ from those of tramadol in several ways. The parent molecule of tramadol inhibits the norepinephrine $(-)$ enantiomer and serotonin reuptake $(+)$ enantiomer, whereas the metabolite (O-desmethyl-metabolite) provides mu receptor activation. ${ }^{15,21}$ Therefore, any opioid-mediated analgesic activity of tramadol is dependent on the amount of metabolized fraction of parent drug. Tramadol is metabolized via the enzyme system cytochrome P450 (CYP) 2D6. Approximately 5\%-15\% of humans are so-called "poor metabolizers", who do not experience satisfactory levels of analgesia with standard doses of tramadol. Unlike tramadol, the analgesic activity of tapentadol resides in the parent molecule, and, CYP2 D6 polymorphism plays no role in determining variations in analgesic response. ${ }^{22}$ This is very important because CYP enzymes contribute to clearance of 
approximately two-thirds of the most commonly prescribed medications, including serotonin or norepinephrine reuptake inhibitors and mu receptor agonists. ${ }^{23,24}$ Tapentadol is metabolized predominantly by $\mathrm{O}$-glucuronidation mediated by UGT1A9 and UGT2B7. ${ }^{25,26}$ The metabolites of tapentadol showed no analgesic activity in animal pain models, ${ }^{10}$ and demonstrated no affinity for the mu receptor or the norepinephrine transporter in binding studies. Following oral administration of tapentadol, 99\% of the parent molecule and its inactive conjugated metabolites which possess no appreciable analgesic activity are excreted via the kidneys. The manufacturer suggests that tapentadol should not be used in severe hepatic or renal failure. There have been no formal studies in these populations. ${ }^{24}$ The bioavailability of orally administered tapentadol is $32 \%$, which is comparable with morphine, but less than methadone, tramadol, or oxycodone. With tapentadol given orally, the time taken to reach peak plasma concentration is $1.25-1.5$ hours. The half-life is 24 hours, and plasma protein binding is $20 \%{ }^{25}$

\section{Extended-release preparation}

The ER tapentadol preparation was specifically developed for the management of moderate-to-severe chronic pain. The controlled release formulation provides a 12-hour duration of activity, as well as the convenience and analgesic uniformity associated with twice per day dosing. The analgesic efficacy of tapentadol is comparable with that of the classic ER opioids, such as controlled-release oxycodone. Opioids have efficacy in inflammatory, somatic, and neuropathic pain conditions, ${ }^{42}$ as well as cancer pain. Based on several largescale clinical trials, described later in this paper, tapentadol ER is associated with better gastrointestinal tolerability, than the typical extended-duration opioids.

$\mathrm{Mu}$ receptor activation is a secondary mechanism responsible for the analgesic effect of tapentadol. Therefore, with chronic use, there is a relatively low degree of mu receptor activation as compared with potent mu opioids and, correspondingly, a slower onset of tolerance. One study demonstrated that the onset of tolerance with chronic use of tapentadol was significantly delayed as compared with morphine. With morphine treatment, the onset of tolerance was almost immediate, with complete tolerance by day 21 . With tapentadol use, a progressive decline in efficiency was observed until day 18 , followed by a plateau of moderate antinociception and complete tolerance on day 51., ${ }^{9,22}$ It is unclear whether benefits of reduced tolerance and dose escalation will be observed with long-term clinical use.

\section{Clinical investigations Osteoarthritis}

A placebo and active-comparator controlled trial of tapentadol ER was performed in 670 patients with moderate-to-severe chronic pain related to chronic osteoarthritis of the knee..$^{27,28}$ Patients were randomly assigned to 28 days of treatment with tapentadol ER $100 \mathrm{mg}$ or $200 \mathrm{mg}$, oxycodone controlledrelease $20 \mathrm{mg}$, or placebo twice daily. The primary endpoint was average pain intensity over the preceding 24 hours (100 mm on visual analog scale). Patients treated with tapentadol ER reported significantly reduced pain intensity vs placebo $(-8.4 \mathrm{~mm} ; P=0.021)$, and analgesia comparable with that in those treated with oxycodone controlled-release $20 \mathrm{mg}$. More patients rated pain relief with tapentadol ER $200 \mathrm{mg}$ "very good" or "excellent" compared with placebo (48.8\% vs 29.2\%). Gastrointestinal disorders occurred in $23 \%$ of placebo patients, $30 \%$ of patients treated with tapentadol ER $100 \mathrm{mg}, 49 \%$ of patients treated with tapentadol ER $200 \mathrm{mg}$, and $56 \%$ of patients on oxycodone controlledrelease $20 \mathrm{mg}$. Constipation was much less common in the tapentadol ER $100 \mathrm{mg}$ and $200 \mathrm{mg}$ groups than in the oxycodone controlled-release group. Nervous system adverse events such as drowsiness, headache, and somnolence, were reported by $15 \%, 24 \%, 34 \%$, and $43 \%$ of patients on placebo, tapentadol ER $100 \mathrm{mg}$, tapentadol ER $200 \mathrm{mg}$, and oxycodone controlled-release $20 \mathrm{mg}$, respectively.

\section{Chronic low back pain}

A large proportion of patients suffer from low back pain, with many having a predominant neuropathic pain component as well. ${ }^{29}$ The safety and effectiveness of tapentadol ER was evaluated in 958 patients presenting with chronic low back pain. ${ }^{13}$ In a randomized, controlled, blinded fashion, patients with moderate-to-severe pain were titrated over 3 weeks to achieve an effective and tolerable twice daily dose of tapentadol ER 100-250 mg, oxycodone $\mathrm{HCl}$ controlled release $20-50 \mathrm{mg}$, or placebo, and then maintained at that dose for 12 weeks.

Additional dose adjustments were allowed to maintain an optimal balance of efficacy and tolerability. Efficacy was measured as change in mean pain intensity from baseline to week 12. Two hundred and thirty-five patients in the tapentadol ER group and 199 patients in the oxycodone CR group entered the 12-week maintenance period. During this time interval, the average 24-hour dose for tapentadol ER was $400 \mathrm{mg}$, and for the oxycodone CR dose was $80 \mathrm{mg}$. Patients treated with tapentadol ER benefited from 
significantly greater reductions in average pain intensity than those on placebo. A higher percentage of patients completed treatment with tapentadol ER (54.1\%) than with oxycodone CR (43.3\%), mainly because of the lower rate of discontinuation due to gastrointestinal side effects $(16.7 \%$ with tapentadol ER vs $32.3 \%$ with oxycodone $C R$ ). The authors concluded that tapentadol ER 100-250 mg twice daily relieved moderate-to-severe chronic low back pain more effectively than placebo, and with fewer adverse event-related discontinuations than oxycodone CR 20-50 mg twice daily.

\section{Diabetic neuropathy pain}

The effectiveness of tapentadol ER in managing moderateto-severe chronic pain was also evaluated in patients with diabetic peripheral neuropathy. This randomized, doubleblind trial involved diabetic patients aged 18 years or older with a diagnosis of moderate-to-severe painful diabetic peripheral neuropathy and symptoms for a minimum of 6 months. During the 3-week, open-label period, 588 patients were titrated to an optimal dose of tapentadol ER 100-250 mg twice daily. The majority of these patients (79.4\%) had a pain intensity rating $\geq 6$ on an 11-point numeric rating scale. Patients were then advanced to the double-blind phase of the trial which consisted of a 12-week maintenance period, during which time patients continued on their study medication. A total of 395 patients who received tapentadol ER in the openlabel period and began the double-blind phase of treatment had a mean decrease in pain intensity from 7.3 indicating severe pain to 3.5 indicating mild pain during open-label treatment. During the double-blind period, the tapentadol ER group had an average pain intensity that remained relatively constant, while the placebo group had a pain intensity that increased in severity $(P<0.001)$.

During the open-label treatment phase, $20.1 \%$ of patients experienced the onset of one or more adverse events and discontinued the study. Following advancement to the double-blind phase, $11.2 \%$ of patients in the tapentadol ER group and $5.7 \%$ of patients in the placebo group discontinued due to adverse events. The most common adverse events were gastrointestinal and led to discontinuation in $10 \%$ of patients. ${ }^{30}$

The finding that tapentadol effectively suppressed neuropathic pain, while a mu receptor agonist was ineffective, may be related to the dual analgesic mechanism associated with this molecule. Agents that have a similar combined mechanism have been shown to be effective in the treatment of neuropathic pain conditions and, at the same time, are able to potentiate the opioid analgesics. Neuropathic pain is usually managed with tricyclic antidepressants, selective serotonin/norepinephrine reuptake inhibitors, anticonvulsants, antiarrhythmics, $N$-methyl-D-aspartate antagonists, and topical therapies. The antinociceptive, antiallodynic, and antihyperalgesic properties of tapentadol have been demonstrated in different rat models of acute and chronic pain. The models have included tail flick, hot plate, writhing, mustard oil colitis, chronic constriction injury, Randall Selitto test, and spinal nerve ligation. ${ }^{31,32}$ The efficacy of norepinephrine inhibitors, such as tricyclic and tetracyclic antidepressants, has been demonstrated in neuropathic pain models. $^{14}$

\section{Long-term safety in patients with chronic pain}

The long-term safety and efficacy of tapentadol ER was evaluated in a large, randomized, open-label trial of patients with chronic pain. ${ }^{33}$ A total of 1117 patients were randomized in a 4:1 ratio to receive twice-daily doses of tapentadol ER $50 \mathrm{mg}$ or oxycodone CR $10 \mathrm{mg}$, which was increased over a 3-day period to tapentadol $100 \mathrm{mg}$ twice daily or oxycodone $20 \mathrm{mg}$ twice daily. Patients were maintained on these analgesics for the next 51 weeks, although dose escalations or reductions were permitted. Additional breakthrough pain was treated with acetaminophen in both treatment groups. Over the 1-year study period, reductions in average pain intensity scores and total daily analgesic dose were stable in both groups.

Patients treated with tapentadol ER reported a lower overall incidence of treatment-related adverse gastrointestinal events, such as nausea, vomiting, and constipation. A lower proportion of patients treated with tapentadol ER experienced pruritus, but a greater number reported headache. Treatmentrelated adverse events led to discontinuation in $22 \%$ of patients in the tapentadol ER group and in $36.8 \%$ of patients in the oxycodone CR group. ${ }^{2}$ Tapentadol ER has been shown to be effective for the management of moderate-to-severe chronic osteoarthritis-related knee pain. Despite the fact that oxycodone CR was shown to be a more potent medication than tapentadol ER with a 5:1 conversion rate $(50 \mathrm{mg}$ tapentadol ER to $10 \mathrm{mg}$ oxycodone CR), a significantly higher proportion of patients in the tapentadol ER group (32\%, $P=0.027$ ) achieved more than $50 \%$ pain relief as compared with the oxycodone CR group $(17.3 \%, P=0.023) .{ }^{4}$ In another study of more than 320 patients, both tapentadol ER and oxycodone CR provided at least a three-point drop on a $0-10$ pain scale in patients with lower back pain. ${ }^{13}$ 
Another study showed that the efficacy of tapentadol ER was comparable with that of oxycodone $\mathrm{CR}(P<0.001) .{ }^{11}$ Tapentadol ER was effective in the treatment of pain secondary to diabetic neuropathy, with $60.5 \%$ of patients reporting at least a $30 \%$ improvement in pain intensity and $34.8 \%$ at least a $50 \%$ improvement, ${ }^{43}$ as compared with data from three randomized, 15-week, double-blind, randomized, Phase III studies, ie, $39.7 \%$ and $27.0 \%$ in chronic low back pain patients, ${ }^{3} 43 \%$ and $32 \%$ in patients with osteoarthritic knee pain, ${ }^{4}$ and $41.3 \%$ and $30.1 \%$ in those with lower back pain and osteoarthritic knee pain. ${ }^{11,16,34-39}$

Opioids have demonstrated analgesic effects comparable with those of tricyclics and gabapentin in the treatment of diabetic peripheral neuropathy. However, tolerability remains a significant issue limiting their use..$^{37,40-46}$ Therefore, opioids are considered a second-line treatment for neuropathic pain. ${ }^{30,47}$ Constipation affects an estimated $40 \%-95 \%$ of patients on long-term opioid therapy. ${ }^{36}$ Opioid-induced constipation rarely improves with time and can be difficult to manage with laxatives. ${ }^{48}$

Clinical studies with tapentadol ER showed that gastrointestinal side effects are of major importance. The incidence of nausea, vomiting, and constipation were $20.1 \%, 9.1 \%$, and $13.8 \%$ in a 15 -week study of tapentadol ER in low back pain, ${ }^{13}$ and $18.1 \%, 7.0 \%$, and $22.6 \%$ in a 1 -year study of patients with chronic low back pain and osteoarthritis pain, ${ }^{49}$ and $21.4 \%, 8.0 \%$, and $10.7 \%$ in patients with painful diabetic polyneuropathy. ${ }^{40}$

\section{Benefits associated with tapentadol ER}

In clinical trials, tapentadol ER has demonstrated an improved gastrointestinal tolerability profile compared with the comparator, oxycodone $\mathrm{CR}$, at equianalgesic doses..$^{5-8}$ The incidence of adverse gastrointestinal effects was $43 \%$ in the tapentadol ER group and $67.3 \%$ in the oxycodone CR group. ${ }^{4}$ Tapentadol ER used for over 15 weeks provided relief for moderate-to-severe chronic low back pain and had a better gastrointestinal tolerability profile than oxycodone $\mathrm{CR}$, with lower rates for nausea, vomiting, constipation, and pruritus. ${ }^{4,9,13}$ Another study demonstrated a lower incidence of nausea, vomiting, and constipation in the tapentadol ER group despite the fact the treatment duration was substantially longer (268 days with tapentadol ER vs 59 days with oxycodone CR). In addition, the incidence of gastrointestinal effects leading to discontinuation of treatment was 2.5 times higher in the oxycodone group. ${ }^{49}$ The observed lower incidence of adverse gastrointestinal effects is likely related to the dual synergistic mechanism of action of tapentadol ER, where appreciable levels of analgesia are achieved with a lesser degree of mu receptor activation.

The ability of tapentadol ER to suppress neuropathic as well as somatic pain suggests it will provide a broader spectrum of efficacy than the currently available ER analgesics. Opioid agonists have limited clinical effectiveness in the management of moderate-to-severe neuropathic pain and are not considered a first-line therapy, ${ }^{1,27,28,30}$ whereas analgesic medications with a combined mechanism of action, including norepinephrine reuptake inhibition, are effective against chronic pain.

Opioids are reserved for patients with refractory neuropathic pain. ${ }^{28,33,50}$ In addition to that, the use of opioid medications is limited because of the side effects associated with mu receptor activation. ${ }^{14,51,52}$ This implies that the development of a compound combining both mechanisms of action is the way to improve the therapeutic range of opioids. Facilitation of monoaminergic transmission by blocking the reuptake of norepinephrine and/or serotonin in the descending pain inhibitory pathways ${ }^{53}$ of the spinal cord is efficacious in the treatment of chronic pain conditions and can enhance the analgesic effects of morphine. ${ }^{52}$

\section{Adverse events}

ER opioids, including tapentadol ER, are associated with a high incidence of side effects and treatment discontinuation. Major adverse effects reported in the Phase II and III trials of tapentadol ER were gastrointestinal intolerance and central nervous system effects, ${ }^{1}$ including nausea, anxiety, diarrhea, and dizziness. ${ }^{40} \mathrm{~A}$ review of the literature shows that the leading side effects of tapentadol are gastrointestinal, with nausea being the most common (30\%), followed by vomiting (18\%), somnolence (15\%), and dizziness (24\%). Significant changes in blood pressure have not been reported in the literature as a side effect of tapentadol ER. Existing norepinephrine reuptake inhibition may potentially have cardiovascular effects, and more research in this area is necessary to determine if there are any significant cardiovascular effects. The literature on the tapentadol ER trials has not shown any vision-related side effects. Because tapentadol is a weak mu agonist, there is a potential for it to activate mu receptors in the Edinger-Westphal nucleus of the midbrain, resulting in a predominance of parasympathetic tone over sympathetic tone that, in turn, leads to myosis and accommodation. More research is warranted to determine if there are any significant effects on vision. 


\section{Contraindications}

While not observed in controlled trials, tapentadol ER, like other extended-duration opioids, may be associated with significant respiratory depression, and should not be administered to patients with acute or severe bronchial asthma or hypercapnia in unmonitored settings or in the absence of resuscitative equipment. Tapentadol ER should also not be used by patients who are receiving monoamine oxidase inhibitors or who have taken them within the previous 14 days due to potential additive effects on norepinephrine levels which may result in adverse cardiovascular events. ${ }^{1,13}$ Tapentadol ER has the potential to precipitate serotonin syndrome and induce physical/ psychological dependence. ${ }^{9}$ Patients at risk of developing serotonin syndrome should not be treated with tapentadol. The development of a potentially life-threatening serotonin syndrome may occur with use of selective norepinephrine reuptake inhibitors, including tapentadol, particularly with concomitant use of serotonergic drugs, such as selective serotonin reuptake inhibitors, selective norepinephrine reuptake inhibitors, tricyclic antidepressants, monoamine oxidase inhibitors, and triptans, and drugs which impair metabolism of serotonin (including monoamine oxidase inhibitors). Serotonin syndrome may include changes in mental status (eg, agitation, hallucinations, and coma), autonomic instability (eg, tachycardia, labile blood pressure, and hyperthermia), and neuromuscular aberrations (eg, hyperreflexia and incoordination). Because tapentadol has effects on norepinephrine reuptake, patients may experience a monoamine syndrome of poorly characterized irritability and agitation, which resembles serotonin syndrome but is not associated with major complications. ${ }^{1,13}$

Tapentadol has an abuse potential similar to that of other potent opioid agonists and is subject to criminal diversion. It is unclear if the taper-resistant preparation will limit diversion, adulteration, and abuse. After 360 days of continuous administration, abrupt discontinuation of tapentadol was associated with mild to moderate withdrawal symptoms.

Based on tapentadol ER trials, dosing may range from $100 \mathrm{mg}$ from $200 \mathrm{mg}$ twice daily, or higher. No dosage adjustment is needed in mild or moderate renal impairment. Tapentadol may be provided to opioid-dependent patients. Data taken from the 1-year safety trial, in which some patients had been taking opioids prior to enrollment, suggests that tapentadol doses of $200 \mathrm{mg}$ or higher every 12 hours may be appropriate. ${ }^{33}$ Both formulations of tapentadol, ie, immediate-release and ER, can be directly converted into equivalent or approximately equivalent total daily doses that provide comparable efficacy and safety. It is anticipated that tapentadol ER will be released as a Schedule II opioid analgesic. In an attempt to limit abuse, tapentadol ER will be formulated as a tamper-resistant tablet that cannot be easily adulterated. The tablet is designed to provide a high degree of mechanical resistance, such as crushing or chewing. ${ }^{4}$

\section{Conclusion}

Tapentadol ER is a novel analgesic with a combined mechanism of action and clinical effectiveness comparable with that of commonly used medications. Based on preliminary clinical trials, tapentadol ER may offer an advance over standard controlled-release opioid preparations in that it offered improved gastrointestinal tolerability, less nausea and vomiting, and less constipation in the osteoarthritis and chronic low back pain efficacy trials than did oxycodone CR. ${ }^{28,30,33}$ It is unclear whether improvements in tolerability may reduce discontinuation of and greater patient compliance with therapy. Clearly, additional longer-term clinical trials are required to verify whether gastrointestinal side effects are lower with tapentadol than with the traditional opioids, such as morphine, hydromorphone, transdermal fentanyl, and methadone.

Initial trials demonstrating efficacy in neuropathic pain suggest that the dual analgesic effects of tapentadol may provide a broader analgesic spectrum than typical opioid analgesics. The pharmacokinetic profile of tapentadol has a low potential for metabolic variation due to enzyme polymorphism, drug-drug interaction, and drug accumulation. Tapentadol has never been studied in patients with chronic liver or renal disease, or in those who are pregnant or elderly. ${ }^{16}$ Tapentadol will need to be rigorously tested in chronic neuropathic pain, cancer-related pain, and cancer-related neuropathic pain.

\section{Disclosure}

The authors report no conflicts of interest in this work.

\section{References}

1. Wade WE, Spruill WJ. Tapentadol hydrochloride: a centrally acting oral analgesic. Clin Ther. 2009;31(12):2804-2818.

2. Etropolski MS, Okamoto A, Shapiro DY, Rauschkolb C. Dose conversion between tapentadol immediate and extended release for low back pain. Pain Physician. 2010;13(1):61-70.

3. Kleinert R, Lange C, Steup A, Black P, Goldberg J, Desjardins P. Single dose analgesic efficacy of tapentadol in postsurgical dental pain: the results of a randomized, double-blind, placebo-controlled study. Anesth Analg. 2008;107(6):2048-2055. 
4. Afilalo M, Etropolski MS, Kuperwasser B, et al. Efficacy and safety of tapentadol extended release compared with oxycodone controlled release for the management of moderate to severe chronic pain related to osteoarthritis of the knee: a randomized, double-blind, placeboand active-controlled phase III study. Clin Drug Investig. 2010; 30(8):489-505.

5. Stegmann JU, Weber H, Steup A, Okamoto A, Upmalis D, Daniels S. The efficacy and tolerability of multiple-dose tapentadol immediate release for the relief of acute pain following orthopedic (bunionectomy) surgery. Curr Med Res Opin. 2008;24(11):3185-3196.

6. Daniels SE, Upmalis D, Okamoto A, Lange C, Häeussler J. A randomized, double-blind, phase III study comparing multiple doses of tapentadol IR, oxycodone IR, and placebo for postoperative (bunionectomy) pain. Curr Med Res Opin. 2009;25(3):765-776.

7. Hartrick C, Van Hove I, Stegmann J, Oh C, Upmalis D. Efficacy and tolerability of tapentadol immediate release and oxycodone $\mathrm{HCl}$ immediate release in patients awaiting primary joint replacement surgery for end-stage joint disease: a 10-day, phase III, randomized, doubleblind, active- and placebo-controlled study. Clin Ther. 2009;31(2): 260-271.

8. Hale M, Upmalis D, Okamoto A, Lange C, Rauschkolb C. Tolerability of tapentadol immediate release in patients with lower back pain or osteoarthritis of the hip or knee over 90 days: a randomized, doubleblind study. Curr Med Res Opin. 2009;25(5):1095-1104.

9. Tzschentke TM, Jahnel U, Kogel B, et al. Tapentadol hydrochloride: a next-generation, centrally acting analgesic with two mechanisms of action in a single molecule. Drugs Today (Barc). 2009;45(7):483-496.

10. Terlinden R, Kogel BY, Englberger W, Tzschentke TM. In vitro and in vivo characterization of tapentadol metabolites. Methods Find Exp Clin Pharmacol. 2010;32(1):31-38.

11. Lange B, Kuperwasser B, Okamoto A, et al. Efficacy and safety of tapentadol prolonged release for chronic osteoarthritis pain and low back pain. Adv Ther. 2010;27(6):381-399.

12. Guay DR. Is tapentadol an advance on tramadol? Consult Pharm. 2009; 24(11):833-840.

13. Buynak R, Shapiro DY, Okamoto A, et al. Efficacy and safety of tapentadol extended release for the management of chronic low back pain: results of a prospective, randomized, double-blind, placebo- and active-controlled Phase III study. Expert Opin Pharmacother. 2010; 11(11):1787-1804.

14. Max MB, Lynch SA, Muir J, Shoaf SE, Smoller B, Dubner R. Effects of desipramine, amitriptyline, and fluoxetine on pain in diabetic neuropathy. N Engl J Med. 1992;326(19):1250-1256.

15. Briley M. Clinical experience with dual action antidepressants in different chronic pain syndromes. Hum Psychopharmacol. 2004; 19(Suppl 1):S21-S25.

16. Sloan P. Tapentadol for acute and chronic pain. Expert Opin Pharmacother. 2010;11(11):1783-1785.

17. Prommer EE. Tapentadol: an initial analysis. J Opioid Manag. 2010; 6(3):223-226.

18. Epstein DH, Preston KL, Jasinski DR. Abuse liability, behavioral pharmacology, and physical-dependence potential of opioids in humans and laboratory animals: lessons from tramadol. Biol Psychol. 2006; 73(1):90-99.

19. Grond S, Sablotzki A. Clinical pharmacology of tramadol. Clin Pharmacokinet. 2004;43(13):879-923.

20. Radbruch L, Grond S, Lehmann KA. A risk-benefit assessment of tramadol in the management of pain. Drug Saf. 1996;15(1):8-29.

21. Gillen C, Haurand M, Kobelt DJ, Wnendt S. Affinity, potency and efficacy of tramadol and its metabolites at the cloned human mu-opioid receptor. Naunyn Schmiedebergs Arch Pharmacol. 2000;362(2): 116-121.

22. Tzschentke T, Christoph T, Kögel B, et al. (-)-(1R,2R)-3-(3-dimethylamino-1-ethyl-2-methyl-propyl)-phenol hydrochloride (tapentadol $\mathrm{HCl}$ ): a novel mu-opioid receptor agonist/norepinephrine reuptake inhibitor with broad-spectrum analgesic properties. J Pharmacol Exp Ther. 2007;323(1):265-276.
23. Nagar S, Raffa RB. Looking beyond the administered drug: metabolites of opioid analgesics. J Fam Pract. 2008;57(Suppl 6):S25-S32.

24. Hartrick C, Van Hove I, Stegmann JU, Oh C, Upmalis D. Efficacy and tolerability of tapentadol immediate release and oxycodone $\mathrm{HCl}$ immediate release in patients awaiting primary joint replacement surgery for end-stage joint disease: a 10-day, phase III, randomized, doubleblind, active- and placebo-controlled study. Clin Ther. 2009;31(2): 260-271.

25. Terlinden R, Ossig J, Fliegert F, Lange C, Göhler K. Absorption, metabolism, and excretion of 14C-labeled tapentadol $\mathrm{HCl}$ in healthy male subjects. Eur J Drug Metab Pharmacokinet. 2007;32(3):163-169.

26. Kneip C, Terlinden R, Beier H, Chen G. Investigations into the drugdrug interaction potential of tapentadol in human liver microsomes and fresh human hepatocytes. Drug Metab Lett. 2008;2(1):67-75.

27. Schmidt CO, Schweikert B, Wenig CM, et al. Modelling the prevalence and cost of back pain with neuropathic components in the general population. Eur J Pain. 2009;13(10):1030-1035.

28. McDonald AA, Portenoy RK. How to use antidepressants and anticonvulsants as adjuvant analgesics in the treatment of neuropathic cancer pain. J Support Oncol. 2006;4(1):43-52.

29. Freynhagen R, Baron R, Gockel U, Tölle TR. painDETECT: a new screening questionnaire to identify neuropathic components in patients with back pain. Curr Med Res Opin. 2006;22(10):1911-1920.

30. Dworkin RH, O'Connor AB, Backonja M, et al. Pharmacologic management of neuropathic pain: evidence-based recommendations. Pain. 2007;132(3):237-251.

31. Tzschentke TM, Christoph T, Kögel B, et al. (-)-(1R,2R)-3-(3-dimethylamino-1-ethyl-2-methyl-propyl)-phenol hydrochloride (tapentadol $\mathrm{HCl}$ ): a novel mu-opioid receptor agonist/norepinephrine reuptake inhibitor with broad-spectrum analgesic properties. J Pharmacol Exp Ther. 2007;323(1):265-276.

32. Monory K, Greiner E, Sartania N, et al. Opioid binding profiles of new hydrazone, oxime, carbazone and semicarbazone derivatives of 14-alkoxymorphinans. Life Sci. 1999;64(22):2011-2020.

33. Maizels M, McCarberg B. Antidepressants and antiepileptic drugs for chronic non-cancer pain. Am Fam Physician. 2005;71(3):483-490.

34. Adams EH, Chwiecko P, Ace-Wagoner Y, et al. A study of AVINZA (morphine sulfate extended-release capsules) for chronic moderateto-severe noncancer pain conducted under real-world treatment conditions - the ACCPT Study. Pain Pract. 2006;6(4):254-264.

35. Avouac J, Gossec L, Dougados M. Efficacy and safety of opioids for osteoarthritis: a meta-analysis of randomized controlled trials. Osteoarthritis Cartilage. 2007;15(8):957-965.

36. Benyamin R, Trescot AM, Datta S, et al. Opioid complications and side effects. Pain Physician. 2008;11(Suppl 2):S105-S120.

37. Gimbel JS, Richards P, Portenoy RK. Controlled-release oxycodone for pain in diabetic neuropathy: a randomized controlled trial. Neurology. 2003;60(6):927-934.

38. Kalso E, Edwards JE, Moore RA, McQuay HJ. Opioids in chronic non-cancer pain: systematic review of efficacy and safety. Pain. 2004; 112(3):372-380.

39. Kivitz A, Ma C, Ahdieh H, Galer BS. A 2-week, multicenter, randomized, double-blind, placebo-controlled, dose-ranging, phase III trial comparing the efficacy of oxymorphone extended release and placebo in adults with pain associated with osteoarthritis of the hip or knee. Clin Ther. 2006;28(3):352-364.

40. Schwartz S, Etropolski M, Shapiro DY, et al. Safety and efficacy of tapentadol ER in patients with painful diabetic peripheral neuropathy: results of a randomized-withdrawal, placebo-controlled trial. Curr Med Res Opin. 2011;27(1):151-162.

41. Argoff CE, Cole BE, Fishbain DA, Irving GA. Diabetic peripheral neuropathic pain: clinical and quality-of-life issues. Mayo Clin Proc. 2006;81(Suppl 4):S3-S11.

42. Eisenberg E, McNicol ED, Carr DB. Efficacy and safety of opioid agonists in the treatment of neuropathic pain of nonmalignant origin: systematic review and meta-analysis of randomized controlled trials. JAMA. 2005;293(24):3043-3052. 
43. Finnerup NB, Otto M, McQuay HJ, Jensen TS, Sindrup SH. Algorithm for neuropathic pain treatment: an evidence based proposal. Pain. 2005; 118(3):289-305.

44. Harati Y, Gooch C, Swenson M, et al. Double-blind randomized trial of tramadol for the treatment of the pain of diabetic neuropathy. Neurology. 1998;50(6):1842-1846.

45. Watson CP, Babul N. Efficacy of oxycodone in neuropathic pain: a randomized trial in postherpetic neuralgia. Neurology. 1998;50(6): $1837-1841$.

46. Watson CP, Moulin D, Watt-Watson J, Gordon A, Eisenhoffer J. Controlled-release oxycodone relieves neuropathic pain: a randomized controlled trial in painful diabetic neuropathy. Pain. 2003;105(1-2): 71-78.

47. Attal N, Cruccu G, Haanpää M, et al. EFNS guidelines on pharmacological treatment of neuropathic pain. Eur J Neurol. 2006;13(11): 1153-1169.
48. Pappagallo M. Incidence, prevalence, and management of opioid bowel dysfunction. Am J Surg. 2001;182(Suppl 5A):11S-18S.

49. Wild JE, Grond S, Kuperwasser B, et al. Long-term safety and tolerability of tapentadol extended release for the management of chronic low back pain or osteoarthritis pain. Pain Pract. 2010;10(5):416-427.

50. Veves A, Backonja M, Malik RA. Painful diabetic neuropathy: epidemiology, natural history, early diagnosis, and treatment options. Pain Med. 2008;9(6):660-674.

51. Martin TJ, Eisenach JC. Pharmacology of opioid and nonopioid analgesics in chronic pain states. J Pharmacol Exp Ther. 2001;299(3): $811-817$.

52. Ossipov MH, Malseed RT, Goldstein FJ. Augmentation of central and peripheral morphine analgesia by desipramine. Arch Int Pharmacodyn Ther. 1982;259(2):222-229.

53. Millan MJ. Descending control of pain. Prog Neurobiol. 2002;66(6): $355-474$.

\section{Publish your work in this journal}

The Journal of Pain Research is an international, peer-reviewed, open access, online journal that welcomes laboratory and clinical findings in the fields of pain research and the prevention and management of pain. Original research, reviews, symposium reports, hypothesis formation and commentaries are all considered for publication.

\section{Dovepress}

The manuscript management system is completely online and includes a very quick and fair peer-review system, which is all easy to use. Visit http://www.dovepress.com/testimonials.php to read real quotes from published authors. 